\title{
Stable pressure driving system for generation of droplets on demand
}

\author{
Ruichang Ke, Limin Lu, Dan Mo, Shenglan Zeng, Binyu Chen and Quanjun Liu \\ State Key Laboratory of Bioelectronics, School of Biological Science and Medical Engineering, \\ Southeast University, Nanjing 210096, PR China. \\ *Quanjun Liu Corresponding author E-mail: Iqj@seu.edu.cn
}

Keywords: Microfluidic; stable pressure; Y-junction chip; droplet; embedded system

Abstract. A stable pressure driving system was designed in this paper, which can be used in Microfluidic Chips to driving reagents. Herein, two embedded controller units are used to control the stability of the reagent bottles pressure, for driving the reagents flow steady on the microfluidic chips. All parameters of system can be set by a friendly upper computer interface, and the upper and lower computer interactive communication via Wi-Fi. Y-junction microfluidic chip was taken to generate water-in-oil droplets that can be observed by Microfluidic Optical Detection Platform. Through the reagent bottles pressure control waveforms and the distribution of droplets sizes to verify the stability and reliability of stable pressure driving system. This convenient and practical system can be widely used in the reagents driving of Microfluidic Chips, and easy to implement automation may one day help enable the realization of the intelligent laboratory.

\section{Introduction}

Microfluidic Chip also called Micro Total Analysis Systems ( $\mu$ TAS) or Lab-on-Chip (LOC) -integrating all the functions of chemical and biological laboratory including sample preparation, reaction, separation, detection etc on a system of a few square centimeters - is becoming more and more widespread. Droplet-based microfluidic chip, one branch of microfluidic chip, is a novel operation small volume liquid technology, becoming an attractive technology in various areas of biology[1], chemistry[2] and chemical biology[3]. In micro-scale channels, the dispersed phase and continuous phase are forced forward and then the dispersed phase will be divided into tiny volumes (micro- or nano-liter), discrete and independent droplets by the interaction between flow shear and surface tension at the junction. Droplet-Based microfluidic chip with dispersed droplet unit as micro reactor can offer many advantages including quick analysis results, low consumption of reagent, high throughput and prevent the droplet evaporation, in addition to avoiding cross contamination between droplets. There is no reason why droplet-Based microfluidic chips are widely used in digital PCR[4], chemical synthesis[5], micro-extraction[6], protein analysis[7], etc.

In the application of microfluidic chip, a high quality, stable driving system is necessary. Traditional microfluidic chip driving system has the electro-wetting[8] driving system, piezoelectric actuators[9] driving system, syringe pumps[10] driving system. And with the development of science and technology, more and more instruments are developing to the direction of miniaturization, portable and intelligence, the laboratory is also becoming intelligent. This requires laboratory instruments in addition to its miniaturization, portable and intelligence can easily access the Internet of Things, and coordinate work with other instruments under the Internet of Things. Therefore it can reduce the investment of human, improve the efficiency of the laboratory. In this paper, we design a new driving system (Stable Pressure Driving System) based on stable air pressure. Compared with the common injection pump driving system, the pneumatic driving systems are easier to implement automation and intellectualization.

\section{Experiment}

The stable pressure driving system consists of two subsystems: (1) Compressed nitrogen generation and pressure control subsystem (pneumatic subsystem), (2) Microfluidic chip subsystem. The stable pressure driving system is showed as Fig. 1a. By opening pressure reducing valve, aerating two reagent 
bottles respectively and maintaining a stable air pressure, which can drive reagent with a steady flow velocity. Pressure control module can change the two reagent bottles' pressure value, which can produce different velocities, also different size of droplets. The reagent bottles pressure are derived from compressed nitrogen bottle and stabilized by a feedback control system with a precision of \pm 5 mbar. The system can be easily extended to have multiple reagent bottles for multichannel microfluidic system application if required.
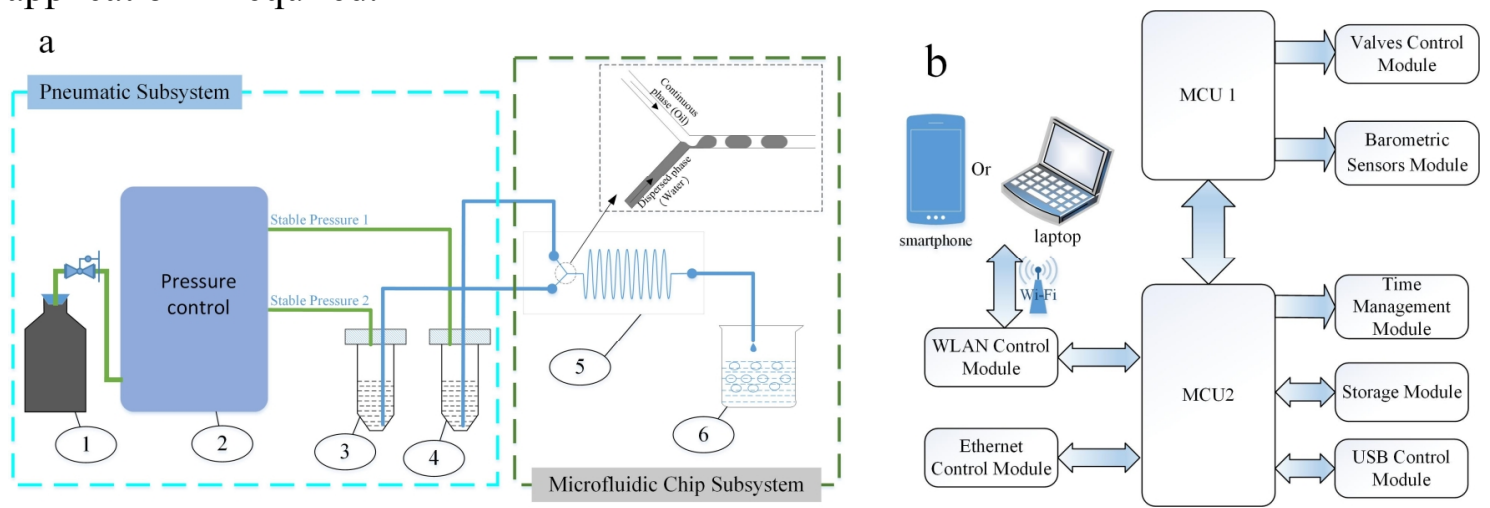

Fig. 1. The stable pressure driving system blocks diagram and hardware control modules blocks diagram. (a) System blocks diagram. The numbers 1-6 of (a) are the compressed nitrogen bottle, pressure control mudule, reagent bottle A, reagent bottle B, Y-junction microfluidic chip, droplets reservoir. Inset: the structure of Y-junction. (b) Hardware control modules blocks diagram.

The pneumatic subsystem is designed to generate two different stable pressures for giving two different reagent flow velocities. The pneumatic subsystem contains three parts: (1) Pneumatic parts: The compressed nitrogen bottle, (2) Hardware circuit modules, (3) Software modules, (4) reagent bottles. The Pneumatic parts are to provide high pressure, guide the gas flow and turn on or off the gas flow by reducing valve.

The hardware control modules have two Microcontroller Unit (MCU) cores. The structure of hardware control modules are illustrated in Fig. 1b. MCU 1 is responsible for four tasks: (1) control barometric sensors and receive pressure values by SPI (Serial Peripheral Interface) protocol, (2) control electromagnetic valves, (3) automatically adjust pressure levels in the two reagent bottles, (4) send barometric sensor data to MCU 2. MCU 2 also has four functions: (1) receive commands from a smartphone or laptop via Wi-Fi, (2) control and manage MCU 1, (3) store date, (4) manage time.

The software modules consist of PC software/Android APP and embedded software. The PC software/Android APP providing a friendly Graphical User Interface (GUI) to send commands (such as set pressure values, set times), display data and waveform, analysis of collected data, and give some help information. The embedded software is the main software module.

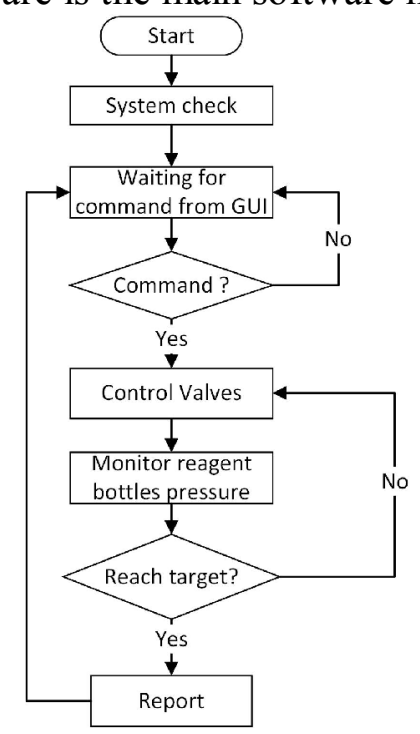

Fig. 2. The soft flow chart of embedded software 
The flow chart of embedded software is shown as Fig. 2. After system checking, MCU 2 waiting commands from GUI via Wi-Fi, and sends data to MCU 1. Then MCU 1 monitors current reagent bottles pressure and through the PID (Proportion Integration Differentiation) algorithm to control the electromagnetic valves and to keep the pressure stability until next pressure commands. Meanwhile, the MCU 1 sends the real-time pressure to the GUI through MCU 2 via Wi-Fi. Through the PID algorithm, the system is faster, more accurate, less gas waste.

Y-junction chip (the width is $500 \mu \mathrm{m}$, the thickness is $1000 \mu \mathrm{m}$ ) is the main part of the microfluidic chip subsystem. Pneumatic subsystem drives two reagent bottles of reagents to the Y-junction chip forming droplets. And Droplet Microfluidic Optical Detection Platform (type: OLYMPUS-IX81) is used to observe the shape of the droplets. In this experiment the dispersed phase is ultrapure water and continuous phase is oil solution (95\% mineral oil and 5\% Span-80).

\section{Results and discussion}

We can use GUI (PC software/Android APP) conveniently to set different pressure values to produce different size of droplets in the application of Microfluidic Chips, and without any other complex operations. The results of experiment are shown in Fig. 3. Statistical histogram in Fig. 3a shows that the size of the droplets mainly concentrated on the range of $0.78 \mathrm{~mm}$ at the 3600 mbar of continuous phase and 1400 mbar of dispersed phase. Some droplets' length which is far away from the $0.78 \mathrm{~mm}$ is likely caused by liquid pipeline larger sloshing in the process of the experiment.
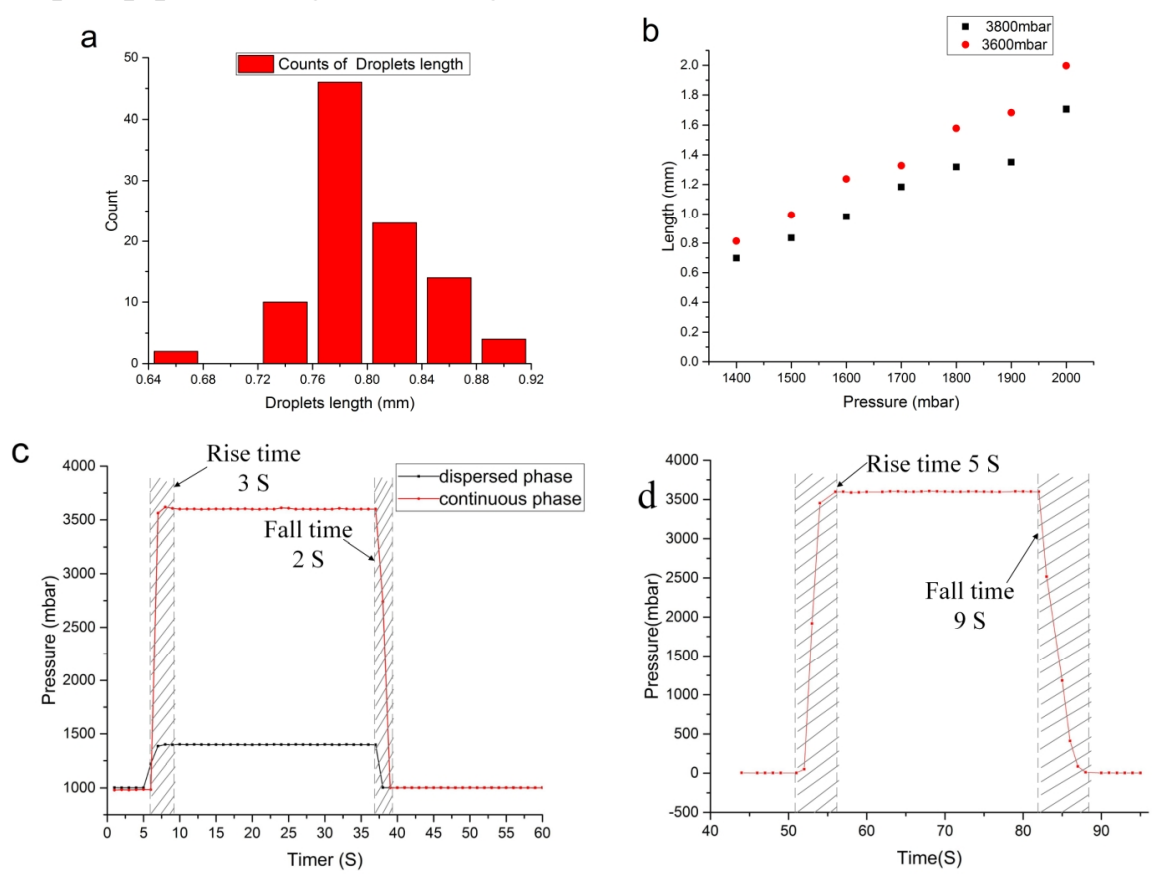

Fig. 3. The result of experiment: (a) The droplets size distribution at the 3600 mbar of continuous phase and $1400 \mathrm{mbar}$ of dispersed phase. (b) The relationship between dispersed phase pressure and droplets length at the $3600 \mathrm{mbar}$ and 3800 mbar of continuous phase. (c)The two reagents bottles pressure control waveforms figure. The pressure of continuous phase bottle is 3600 mbar and the pressure of dispersed phase bottle is 1400 mbar. (d) bottle pressure control waveform of a market pressure driving system. The shadows in (c) and (d) hint the rise and fall time.

The lengths of the droplets appear a linear increase with the increase of dispersed phase pressure, and a lower continuous phase pressure can produce longer droplets in widely range pressure (shown as Fig. 3b). Fig. $3 \mathrm{~b}$ also indicates the system control range is wide, and this wider than traditional driving system, such as syringe pumps driving system.

In The stable pressure driving system, the stability of the two reagent bottles pressure is very important. And the stability of system control can see from the two reagents bottles pressure control waveforms (Fig. 3c). From Fig. 3c, we can see two smooth and steady of pressure waveforms with a precision of \pm 5 mbar and \pm 2 mbar at target pressures are 3600 mbar and 1400 mbar for driving liquid reagents. Compared with the same kind of drive system on the market (shown with shadow in Fig. 3d), 
the system has a fast respond time (shown with shadow in Fig. 3c). Therefore, the stable pressure driving system can be used in some fast respond Microsystem.

\section{Conclusions}

In summary, we have demonstrated a widely range, fast respond, stable, reliable, convenient and practical system. The system can drive reagents stably to produce uniform and fast droplets. We believe that the driving system based on stable air pressure will be widely used in the reagents driving of Microfluidic Chips, and even replace traditional microfluidic chip driving systems in some areas. The stable pressure driving system will have a huge commercial market. In particular, the stable pressure driving system is easier to implement automation may one day help enable the realization of the intelligent laboratory.

\section{Acknowledgment}

This work was supported by National Basic Research Program of China (2011CB707600), the National Natural Science Foundation of China (61071050, 61372031), Tsinghua National Laboratory for Information Science and Technology (TNList), Cross-discipline Foundation and "the Fundamental Research Funds for the Central Universities", and the Foundation for the author of National excellent doctoral dissertation.

\section{References}

[1] Y. Schaerli,F. Hollfelder, "The potential of microfluidic water-in-oil droplets in experimental biology". Mol Biosyst, Vol. 5, 2009, p. 1392-404.

[2] B. J. Thomas, B. A. Harruff-Miller, C. E. Bunker,W. K. Lewis, "Infrared spectroscopy of Mg-CO2 and Al-CO2 complexes in helium nanodroplets". The Journal of chemical physics, Vol. 142, 2015, p. 174310.

[3] D. B. Weibel,G. M. Whitesides, "Applications of microfluidics in chemical biology". Curr Opin Chem Biol, Vol. 10, 2006, p. 584-91.

[4] H. Tanaka, S. Yamamoto, A. Nakamura, Y. Nakashoji, N. Okura, N. Nakamoto, K. Tsukagoshi,M. Hashimoto, "Hands-Off Preparation of Monodisperse Emulsion Droplets Using a Poly(dimethylsiloxane) Microfluidic Chip for Droplet Digital PCR". Anal Chem, Vol. 87, 2015, p. 4134-43.

[5] T. A. Meier, R. J. Beulig, E. Klinge, M. Fuss, S. Ohla,D. Belder, "On-chip monitoring of chemical syntheses in microdroplets via surface-enhanced Raman spectroscopy". Chem Commun (Camb), Vol. 51, 2015, p. 8588-91.

[6] S. Wen, X. Zhu, X. Wu,X. Qin, "Directly suspended droplet microextraction coupled with electrothermal atomic absorption spectrometry for the speciation of chromium(iii)/chromium(vi)". Anal. Methods, Vol. 6, 2014, p. 9777-9782.

[7] L. Ye, X. Wang, J. Han, F. Gao, L. Xu, Z. Xiao, P. Bai, Q. Wang,B. Zhang, "Two dimensional separations of human urinary protein digest using a droplet-interfaced platform". Anal Chim Acta, Vol. 863, 2015, p. 86-94.

[8] W. C. Nelson, I. Peng, G. A. Lee,e. al., "Incubated Protein Reduction and Digestion on an Electrowetting-on-Dielectric Digital Microfluidic Chip for MALDI-MS". Anal. Chem., Vol. 82, 2010, p. 9932-9937.

[9] J. Xu,D. Attinger, "Drop on demand in a microfluidic chip". J. Micromech. Microeng, Vol. 18, 2008, p. 065020.

[10] M. Noerholm, H. Bruus, M. H. Jakobsen, P. Telleman,N. B. Ramsing, "Polymer microfluidic chip for online monitoring of microarray hybridizations". Lab on a chip, Vol. 4, 2004, p. 28-37. 